\title{
A comparative study of several bootstrap-based tests for the volatility in continuous-time diffusion models
}

\author{
Tianshun Yan ${ }^{1,2 \mathbb{D}} \cdot$. Liping Zhang ${ }^{2}$
}

Received: 28 July 2017 / Accepted: 28 January 2019 / Published online: 9 February 2019

(C) ISEG - Instituto Superior de Economia e Gestão 2019

\begin{abstract}
This article develops three bootstrap-based tests for a parametric form of volatility function in continuous-time diffusion models. The three tests are the generalized likelihood ratio test by Fan et al. (Ann Stat 29(1):153-193, 2001), the nonparametric kernel test (LWZ) by Li and Wang (J Econometrics 87(1):145-165, 1998) and Zheng (J Econ 75(2):263-289, 1996) and the nonparametric test (CHS) by Chen et al. (2017). Monte Carlo simulations are performed to evaluate the sizes and power properties of these bootstrap-based tests in finite samples over a range of bandwidth values. We find that the bootstrap-based tests are not influenced by prior restrictions on the functional form of the drift function and that the bootstrap-based CHS test has better power performance than the bootstrap-based GLR and LWZ tests in detecting a parametric form of volatility. An empirical study on weekly treasury bill rate is further conducted to demonstrate these bootstrap-based test procedures.
\end{abstract}

Keywords Continuous-time diffusion models - Generalized likelihood ratio test Nonparametric kernel test · Bootstrap · Treasury bill rate

JEL Classification $\mathrm{C} 12 \cdot \mathrm{C} 13 \cdot \mathrm{C} 58$

? Tianshun Yan

yantianshun@aliyun.com

1 School of Mathematics and Statistics, Xi'an Jiaotong University, Xi'an, Shaanxi, China

2 School of Finance, Chongqing Technology and Business University, Chongqing, China 